\title{
Physiology
}

Aukema, H.M. p19

Baeyer, H. von p52

Bailey, M.A. p79

Bakker, W.W. p

Banas, B. p1

Bankovic-Calic, N. p19

Borghuis, T. p1

Burnstock, G. p79

Chen, S.-M. p113

Chraïbi, A. p37

Cooper, M.E. p26

Cox, A.J. p26

Decaux, G. p11

Dimos, I. p99

Eguchi, N. p42

Eguchi, Y. p42

Fan, M. p91

Fehrenberg, C. p52
Field, M.J. p121

Gilbert, R.E. p26

Groneberg, D.A. p52

Grosse-Siestrup, C. p52

Haider, W. p52

Hakamada-Taguchi, R. p42

Hedeshi, A. p11

Ho, L.-T. p113

Horisberger, J.-D. p37

House, J.D. p19

Hus-Citharel, A. p79

Imbert-Teboul, M. p79

Jackson, C.J. p121

Jolma, P. p91

Kähönen, M. p9

Kalliovalkama, J. p91

Kanellis, J. p26

\author{
Kapojos, J.J. p1 \\ Khong, T. p26 \\ Kööbi, P. p91 \\ Kuo, C.-D. p113 \\ Kuypers, D. p65 \\ Levidiotis, V. p26 \\ Liao, J.-F. p113 \\ Marchetti, J. p79 \\ Milner, P. p79 \\ Minami, K. p59 \\ Moilanen, E. p91 \\ Musch, W. p11 \\ Mysliwiec, M. p105 \\ Naesens, M. p65 \\ Nakajima, H. p42 \\ Numabe, A. p42 \\ Oda, H. p42 \\ Ogata, J. p59
}

Ogborn, M.R. p19

Pawlak, D. p105

Pawlak, K. p105

Poelstra, K. p1

Pollock, C.A. p121

Poronnik, P. p121

Pörsti, I. p91

Power, D.A. p26

Qi, W. p121

Quarcoo, D. p52

Sata, T. p5

Schmitt, R. p52

Scholbach, J. p99

Scholbach, T. p99

Segawa, K. p59

Seiki, K. p42

Shigematsu, A. p59

Shiraishi, M. p59
Stacker, S.A. p26

Steels, P. p65

Sung-Teh, K. p59

Tikkanen, I. p91

Tsuchida, T. p42

Turner, C.M. p79

Uehara, Y. p42

Uezono, Y. p59

Unger, V. p52

Unwin, R.J. p79

Urade, Y. p42

Vanrenterghem, Y. p65

Verberckmoes, R. p65

Yamamoto, C. p59

Young, B. p121

\section{Subject Index Vol. 96, 2004}

Adriamycin $\mathrm{p} 42$

Albuminuria $\mathrm{p} 42$

Amiloride p37

Angiotensin II p59

Arterial smooth muscle, $\mathrm{AT}_{1}$ receptor blockade p91

Atherosclerosis p105

ATP p79

$\mathrm{AT}_{1}$ receptor $\mathrm{p} 91$

Autologous blood perfusion p52

Bartter's syndrome, pathophysiology p65

Biliary zinc secretion p113

Cardiovascular disease p105

CC chemokines p105

Colforsin daropate hydrochloride p59

Conductance vessels p91
Diuresis p11

Ecto-ADPase p1

Endothelium p1

Epithelial sodium channel p37

Extracellular nucleotides p79

Fibroblast p121

Fibrosis p19

Gitelman's syndrome, pathophysiology p65

Glomerulonephritis p26

Healthy children, tissue perfusion p99

Hemopexin $\mathrm{p} 1$

Hyponatremia p11

Intestinal zinc absorption p113

Isolated kidney, preservation solutions $\mathrm{p} 52$

Lipopolysaccharide p1
Matrix metalloproteinases p12

Mesangium $\mathrm{p}$

Metallothionein p113

Natriuresis p1 1

Nephritis $p 42$

Noradrenaline p59

Ochratoxin A p19

Oxidative stress p105

Phosphoinositide signalling pathways $\mathrm{p} 19$

Pig model, nephropathy p19

Plasminogen activator inhibitor p121

Potassium channels p91

P2 receptors, rat glomerulus p79

Preservation solutions, porcine kidney $\mathrm{p} 52$

Prostaglandin D synthase p42
Protease $\mathrm{p} 37$

Proteinuria $\mathrm{p} 26$

P2Y receptors p79

Renal blood flow p59

Salt-losing tubulopathies p65

Self-inhibition, extracellular $\mathrm{Na}^{+}$ p37

Sensorineural deafness $\mathrm{p} 65$

SIADH patients, salt depletion p11

Tissue inhibitor matrix metalloproteinases $\mathrm{p} 121$

- perfusion p99

Urea $\mathrm{p} 11$

Uric acid p11

Vascular endothelial growth factor receptor p26

Zinc p113

\section{KARGER}

(C) 2004 S. Karger AG, Basel

Fax + 41613061234

E-Mail karger@karger.ch

www. karger.com
Accessible online at:

www.karger.com/nep 